\title{
PREVALÊNCIA DE COMPULSÃO ALIMENTAR PERIÓDICA EM INDIVÍDUOS DO SUDOESTE DO PARANÁ
}

\author{
Evellyn Claudia Wietzikoski ${ }^{1}$ \\ Daniela Anelli ${ }^{2}$ \\ Samantha Wietzikoski Sato ${ }^{3}$ \\ Lediana Dalla Costa ${ }^{4}$ \\ Vivian Francielle França ${ }^{5}$
}

WIETZIKOSKI, E. C.; ANELLI, D.; SATO, S. W.; COSTA, L. D.; FRANÇA, V. F. Prevalência de compulsão alimentar periódica em indivíduos do sudoeste do Paraná. Arq. Cienc. Saúde UNIPAR, Umuarama, v. 18, n. 3, p, 173-179, set/dez. 2014.

\begin{abstract}
RESUMO: Devido o transtorno de compulsão alimentar periódico estar aumentando significativamente e pela inexistência de estudos na região sudoeste objetivou-se verificar a prevalência deste transtorno em indivíduos do sudoeste do Paraná, por meio da aplicação da Escala de Compulsão Alimentar Periódica (ECAP) validada para o português. Trata-se de um estudo quantitativo realizado por meio de aplicação de um questionário de coleta de dados autoaplicável em dois postos de saúde do sudoeste do Paraná. A pesquisa foi aprovada pelo Comitê de Ética, depois de submetido na Plataforma Brasil, de acordo com a resolução 466/2012 do Conselho Nacional de Saúde. A amostra em estudo foi constituída por 120 (100\%) participantes do sexo feminino em estado nutricional de eutrofia, sobrepeso e obesidade, com idade mínima de 20 e máxima de 59 anos (média \pm EPM de 35,25 $\pm 0,95$ anos), observamos que 80\% da amostra não apresentam compulsão alimentar periódica, $15 \%$ apresentaram compulsão alimentar periódica moderado e $5 \%$ apresentaram compulsão alimentar periódica severa com média de 32,83 pontos. Ao analisar a faixa etária de maior risco, observa-se que os indivíduos entre 20-29 anos e 3039 anos apresentam pontuações mais elevadas $(\mathrm{F}(3,116)=2,47 ; \mathrm{p} \leq 0.05)$. Os indivíduos obesos da faixa etária de 30-39 anos apresentaram escores mais elevados sendo significantes em relação aos indivíduos eutróficos da mesma faixa etária $(\mathrm{p} \leq 0,05)$. Assim conclui-se que o IMC mais elevado é fator de risco para desenvolvimento de TCAP, assim como em indivíduos com menor faixa etária na idade adulta. PALAVRAS-CHAVE: Transtorno alimentar; Compulsão alimentar periódica; Escala compulsão alimentar periódica.
\end{abstract}

\section{PREVALENCE OF PERIODIC BINGE EATING DISORDER IN THE POPULATION IN THE SOUTHWEST OF PARANÁ}

ABSTRACT: Binge eating disorder (BED) is significantly increasing, and the lack of studies in the southwestern region, the aim of this study was to determine the prevalence of BED in subjects in southwestern Paraná by applying the Binge Eating Scale (BES) validated for Portuguese. This is a quantitative study conducted through a self-administered data collection questionnaire applied in two health centers in southwestern Paraná. The study was approved by the Ethics Committee, after undergoing Platform Brazil, according to Resolution 466/2012 from the National Health Council (CNES). The study sample consisted of $120(100 \%)$ female participants in the nutritional status of normal weight, overweight and obesity, with a minimum age of 20 and maximum of 59 years (mean \pm SEM of $35.25 \pm 0.95$ years). It was observed that $80 \%$ of the sample did not have BED, while $15 \%$ presented moderate BED and $5 \%$ had severe BED, averaging 32.83 points. When analyzing the age group presenting the greatest risk, it could be observed that individuals between 20-29 years old and 30-39 years old have the highest scores $(\mathrm{F}(3.116)=2.47, \mathrm{p} \leq 0.05)$. Obese individuals in the age group of 30-39 years old had higher scores, being significant when compared to normal individuals in the same age group $(\mathrm{p} \leq 0.05)$. Thus, it can be concluded that higher body mass index is a risk factor for developing BED, as well as in individuals with lower age in adulthood.

KEYWORDS: Eating disorder; Binge eating; Binge eating scale.

Introdução

O transtorno de compulsão alimentar periódica (TCAP) foi descrito pela primeira vez na década de 50, entretanto, apesar de ser classificado como um transtorno alimentar (TA) sem outra especificação, os seus critérios diagnósticos foram incluídos no apêndice B da DSM-IV (Manual Diagnóstico e Estatístico de Doenças Mentais - Quarta Edição) somente em 1994, como uma nova categoria a ser melhor investigada (AMERICAN PSYCHIATRIC ASSOCIATION, 1994; PAPELBAUM; APPOLINÁRIO, 2001; APPOLINARIO, 2004; AZEVEDO; SANTOS; FONSECA, 2004).

Tais critérios diagnósticos envolvem: 1) a presença de episódios de compulsão alimentar (ECA) recorrentes, caracterizados por ingestão de quantidade excessiva de alimentos associada à sensação de perda de controle; 2) a presença de pelo menos três indicadores de perda de controle (comer mais rápido do que o normal, comer até sentir-se cheio, comer muito mesmo sem estar sentindo fome, comer sozinho por embaraço pelo tanto que ingere e, sentir-se envergonhado, triste ou culpado após o episódio); 3) sentimentos de angústia relacionados à presença dos $\mathrm{ECA}$; 4) frequência e duração média de ECA de dois dias por semana por seis meses; e, 5) não estar associada ao uso regular de mecanismos compensatórios inadequados para controlar o peso (ex. purgação) e não ocorrer na vigência de anorexia nervosa e bulimia nervosa (AMERICAN PSYCHIATRIC ASSOCIATION, 1994; PALAVRAS et al., 2011).

Uma das ferramentas utilizadas para o diagnóstico do transtorno da compulsão alimentar é a escala de compulsão alimentar periódica (ECAP), que permite observar o comportamento alimentar de pacientes em circunstâncias

DOI: https://doi.org/10.25110/arqsaude.v18i3.2014.5193

${ }^{1}$ Doutora em Farmacologia pela Universidade Federal do Paraná. Docente da UNIPAR - Universidade Paranaense. Endereço para correspondência: Rua Higienópolis, 2718 - CEP 85500-000, Umuarama-PR, E-mail: evellyn@unipar.br.

${ }^{2}$ Graduada em Nutrição, Universidade Paranaense - UNIPAR

${ }^{3}$ Mestre em Farmacologia pela Universidade Federal do Paraná. Docente da UNIPAR (Universidade Paranaense de Umuarama).

${ }^{4}$ Mestre em Saúde e Gestão do Trabalho pela UNIVALI. Docente da UNIPAR (Universidade Paranaense de Francisco Beltrão).

${ }^{5}$ Meste em Educação Física pela Universidade Federal do Paraná. Docente do Curso de Nutrição da UNIPAR (Universidade Paranaense de Francisco Beltrão). 
distintas (FREITAS et al., 2001).

O TCAP pode ocorrer em indivíduos com peso normal, mas é mais comum em indivíduos com sobrepeso ou obesos. A maioria tem uma longa história de repetidas tentativas de fazer dietas e sentem-se desesperados com sua dificuldade de controlar a ingestão de alimentos. Alguns continuam tentando restringir o consumo de calorias, enquanto outros abandonam quaisquer esforços de fazer dieta, em razão de fracassos repetidos (SPITZER et al., 1993). Estima-se que consumo nos ECA chega aproximadamente $5.000 \mathrm{kcal}$ podendo variar a gravidade dos episódios conforme aumenta os valores do Índice de Massa Corpórea (IMC) (PASSOS; STEFANO; BORGES, 2005).

A terapia nutricional tem como objetivo diminuir as compulsões, regularizar e criar um padrão de refeições, proporcionarem uma maior variedade de alimentos para a dieta, corrigir deficiências nutricionais e estabelecer práticas de alimentação saudável (LATTERZAR et al., 2004; SALDAÑA, 2001). Assim, a ECAP pode ser uma ferramenta útil para identificar pacientes de risco e a partir disto auxiliar uma abordagem para propor um tratamento precoce.

Portanto, em virtude de ser um transtorno alimentar que vem aumentando significativamente e não existirem dados da existência de estudos relacionando o nível de prevalência de TCAP em pessoas do sudoeste do Paraná, decidiu-se desenvolver esse trabalho, cujo objetivo foi verificar a prevalência de TCAP em indivíduos do sudoeste do Paraná, através da aplicação da ECAP, traduzida para o português e validada por Freitas et al. (2001).

\section{Material e Método}

\section{Tipo de estudo}

Trata-se de um estudo quantitativo, que foi realizado por meio de aplicação de um questionário de coleta de dados autoaplicável, previamente traduzido e validado por Freitas et al. (2001), com linguagem acessível ao entendimento da média da população estudada. O presente estudo foi submetido à análise do Comitê de Ética em Pesquisa da Universidade Paranaense (UNIPAR) e Plataforma Brasil, de acordo com a resolução 466/2012 do Conselho Nacional de Saúde (CNES) e aprovado pelo parecer consubstanciado ${ }^{\circ}$ 300.922, de 13/06/2013.

\section{Local}

Dois postos de saúde localizados nos municípios do sudoeste do Paraná foram escolhidos para lócus dessa pesquisa. Nestes postos de saúde não são realizados atendimentos pelo nutricionista devido à ausência de vagas de trabalho para este profissional. Dessa forma, dados sobre o estado nutricional da população do sudoeste são desconhecidos.

\section{Critérios de inclusão}

Foram incluídos no estudo participantes do sexo feminino, com faixa etária entre 20 a 59 anos, agrupados de acordo com o estado nutricional sendo eutrofia (IMC 18,5$24,9 \mathrm{~kg} / \mathrm{m}^{2}$ ), sobrepeso (IMC $25,0-29,9 \mathrm{~kg} / \mathrm{m}^{2}$ ) e obesidade (IMC $\geq 30 \mathrm{~kg} / \mathrm{m}^{2}$ ) e que aceitarem participar voluntariamente da pesquisa, assinando o termo de consentimento livre e es- clarecido (TCLE).

Critério de exclusão: Foram excluídos do estudo indivíduos do sexo feminino que não se encontravam na faixa etária determinada pelos critérios de inclusão e que não estavam de acordo com IMC estabelecido. Também foram excluídos gestantes, lactantes, indivíduos em estado de desnutrição, indivíduos do sexo masculino, crianças ou aquelas que não aceitarem assinar o TCLE.

Amostra: Este estudo foi realizado em uma amostra de 120 participantes, que se enquadraram nos critérios de inclusão e que concordaram em participar deste estudo. A coleta de dados foi realizada por conveniência e em participantes considerados acessíveis e dispostos a participar da pesquisa (BARBETTA, 2012).

Avaliação antropométrica: Para determinação do IMC, o peso foi mensurado em pé, descalço e com o mínimo de roupa possível, posiciona-se no centro do equipamento, ereto, com os pés juntos e os braços estendidos ao longo do corpo. Para a estatura é utilizado um estadiômetro fixo, conta o qual os indivíduos se posicionaram, em posição ortostática, com os pés e os calcanhares unidos, encostado á parede, mensurando-se a maior distância entre a região plantar e o vértex. Em seguida foi realizado o calculo do IMC, sendo o $\mathrm{kg} / \mathrm{m}^{2}$ e classificado de acordo OMS, 1997: Magreza $<18,5$; Eutrofia 18,5 - 24,9; Sobrepeso 25,0 - 29,9; Obesidade $\geq$ 30,0 .

\section{Procedimento metodológico}

A metodologia empregada no estudo foi não invasiva. Para avaliação do TCAP foi aplicado um questionário no período de junho a setembro de 2013. A ECAP foi desenvolvida por Gormally et al. (1982). O instrumento é construído por três etapas. Na primeira etapa são definidas as características da CAP, originando um grupo de 16 perguntas. Dessas, oito referem-se a manifestações comportamentais (p. ex., comer escondido), e oito descrevem sentimentos e cognições (p. ex., sensação de falta de controle depois do episódio) relacionadas à CAP. Numa segunda etapa, são construídas afirmativas que refletem a gravidade de cada característica, e são designados pontos (de 0 a 3). Na terceira etapa, verifica-se a gravidade da CAP: a frequência, a quantidade de comida e o grau de emoção envolvido num episódio de CAP. Essas perguntas compõem uma escala do tipo Likert, constituída por uma lista de 16 itens e 62 afirmativas, das quais deve ser selecionada, em cada item, aquela que melhor representa a resposta do indivíduo. A cada afirmativa corresponde um número de pontos de 0 a 3, abrangendo desde a ausência (" 0 ") até a gravidade máxima ("3") da CAP. O escore final é o resultado da soma dos pontos de cada item (FREITAS et al., 2001).

O somatório do questionário foi classificado de acordo com os 3 escores: Menor ou igual a 17 é considerado sem compulsão alimentar periódica (CAP), 18 a 26 são considerados com CAP moderados e maior ou igual a $27 \mathrm{com}$ CAP grave.

Ao final da conclusão deste trabalho, os participantes do estudo receberam seus escores individuais com suas respectivas avaliações. Foram fornecidos informativos a todos os participantes do estudo, salientando a necessidade de tratamento nutricional, médico e psicológico. Visamos ain- 
da, conscientizar o indivíduo da importância da qualidade de vida, bem como evitando alimentações errôneas adotadas ao estilo de vida.

\section{Análise e tabulação dos dados}

Os dados analisados nos questionários foram digitados e tabulados, sendo realizada uma análise quantitativa e descritiva. Os escores obtidos a partir das somatórias das questões respondidas pelas participantes voluntárias do questionário da ECAP foram analisados por ANOVA, seguidas pelo teste post-hoc de Tukey, e considerado como significante quando o $\mathrm{p} \leq 0,05$.

\section{Resultados}

Características dos indivíduos participantes da pesquisa

A amostra em estudo foi constituída por 120 (100\%) participantes do sexo feminino com idade mínima de 20 e máxima de 59 anos (média \pm EPM de 35,25 $\pm 0,95$ anos), que foram classificadas em quatro grupos de acordo com a faixa etária, conforme descrito na tabela 1.

Tabela 1: Características dos participantes entrevistados no período de junho a setembro de 2013.

\begin{tabular}{ccc}
\hline $\begin{array}{c}\text { Faixa Etária } \\
\text { (anos) }\end{array}$ & $\begin{array}{c}\text { Entrevistados } \\
\text { (n) }\end{array}$ & $\begin{array}{c}\text { Porcentagem } \\
(\mathbf{\%})\end{array}$ \\
\hline $20-29$ & 41 & 34,16 \\
$30-39$ & 38 & 31,67 \\
$40-49$ & 26 & 21,67 \\
$50-59$ & 15 & 12,50 \\
TOTAL & $\mathbf{1 2 0}$ & $\mathbf{1 0 0}$ \\
\hline
\end{tabular}

Previamente a aplicação do questionário foi realizada a análise antropométrica para determinar o estado nutricional dos participantes da pesquisa. De acordo com a OMS, dentre os métodos utilizados atualmente para determinar se uma pessoa está dentro da faixa de normalidade do peso, é o IMC, que é calculado dividindo-se o peso (em $\mathrm{Kg}$ ) do indivíduo por sua altura elevada ao quadrado (WHO, 1995; SANTANA, 2012). São considerados indivíduos eutróficos aqueles cujo valor do IMC está entre 18,5 e $24,9 \mathrm{Kg} / \mathrm{m}^{2}$. Os valores de IMC entre 25 e $29,9 \mathrm{Kg} / \mathrm{m}^{2}$ definem o paciente como portador de sobrepeso e valores igual e acima de 30 estará relacionada à obesidade. Na tabela 2, pode-se observar a média do IMC dos indivíduos entrevistados.

Tabela 2: IMC dos participantes entrevistados no período de junho a setembro de 2013.

$\begin{array}{cccc}\begin{array}{c}\text { Estado } \\ \text { Nutricional } \\ \mathbf{N}=40\end{array} & \begin{array}{c}\text { Peso Kg } \\ (\text { média } \pm\end{array} & \begin{array}{c}\text { Altura m } \\ \text { (média } \pm\end{array} & \begin{array}{c}\text { IMC Kg/m } \\ \text { (média } \pm\end{array} \\ \text { EPM) } & \text { EPM) } & \text { EPM) }\end{array}$

\begin{tabular}{llll}
\hline Eutrófico & $59,20 \pm 1,01$ & $1,62 \pm 0,009$ & $22,41 \pm 0,29$ \\
Sobrepeso & $69,52 \pm 0,91$ & $1,61 \pm 0,009$ & $26,64 \pm 0,23$ \\
Obesidade & $83,18 \pm 1,05$ & $1,60 \pm 0,007$ & $32,06 \pm 0,26$ \\
\hline
\end{tabular}

Avaliação dos escores obtidos por meio da ECAP aplicada aos participantes da pesquisa

Para a interpretação dos resultados, foram quantificados os escores obtidos por cada indivíduo no questionário que identifica se o indivíduo apresenta CAP. Os sujeitos que pontuou valores menores ou iguais a 17 pontos foram considerados indivíduos que não apresentavam TA. O escore de 17 é utilizado como ponto de corte para que se obtenha sensibilidade suficiente e para que o diagnóstico de CAP possa ser confirmado por uma entrevista clínica, trata-se de um questionário de rastreamento (RICCA et al., 2000; FREITAS et al., 2001).

Os sujeitos que pontuaram valores iguais a 18 ou 26 pontos foram considerados indivíduos que apresentavam uma CAP moderada, no qual já é possível observar sinais de comportamentos e atitudes compulsivas em relação à alimentação.

O indivíduo foi considerado com CAP severa, quando pontuou valores acima de 27 pontos. Neste estágio, o paciente apresenta compulsão pela ingestão de grande quantidade de comida em um período de tempo delimitado (até duas horas), acompanhado da sensação de perda de controle sobre o que ou o quanto se come de forma grave.

Na tabela 3 observou-se que $80 \%$ da amostra apresentaram valor menor ou igual a 17 pontos, ou seja, não apresentam CAP. A média de CAP encontrada foi de 7,27 pontos. Dos entrevistados, $15 \%$ apresentaram CAP moderado, no qual a média de pontos encontrada foi de 21,27 pontos e $5 \%$ apresentaram CAP severa com média de 32,83 pontos.

Tabela 3: Classificação dos indivíduos de acordo com o escore apresentado na ECAP

\begin{tabular}{lcc}
\hline $\begin{array}{c}\text { Classificação da } \\
\text { ECAP }\end{array}$ & $\begin{array}{c}\text { Entrevistados } \\
\text { (n) }\end{array}$ & Percentual (\%) \\
\hline Ausência CAP & 96 & 80 \\
CAP moderado & 18 & 15 \\
CAP severo & 6 & 5 \\
TOTAL & $\mathbf{1 2 0}$ & $\mathbf{1 0 0}$ \\
\hline
\end{tabular}

Como é possível observar na figura 1, a ANOVA de uma via $(\mathrm{F}(2,117)=6,12 ; \mathrm{p} \leq 0.01)$, seguido pelo post-hoc de Tukey $(\mathrm{p} \leq 0,01)$ revelou que os indivíduos com obesidade apresentaram escores mais elevados que foram significativos em relação ao grupo eutrófico. Apesar disso, observa-se que todos os grupos, independente do estado nutricional não atingiram o ponto de corte de 17 pontos, demonstrando a ausência da CAP. Entretanto, os indivíduos com IMC maior apresentaram uma pontuação na ECAP mais elevada, refletindo maiores riscos na população com obesidade. 


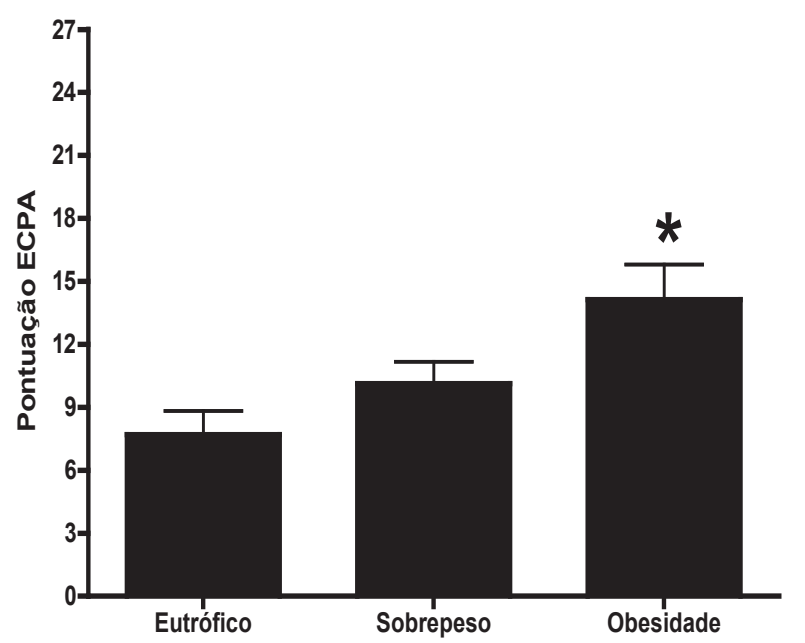

Figura 1: Escore de respostas obtidas na ECAP em indivíduos entrevistados em dois municípios do sudoeste do Paraná agrupados pelo estado nutricional. Os dados expressam a média \pm EPM. ANOVA de uma via, seguida do post hoc de Tukey, ${ }^{*} \mathrm{p} \leq 0,01$, comparado ao grupo eutrófico.

A figura 2 demonstra os resultados obtidos na ECAP em indivíduos agrupados pela faixa etária. Ao analisar a faixa etária de maior risco, observa-se que os indivíduos entre 20-29 anos e 30-39 anos apresentam pontuações mais elevadas. Uma ANOVA de uma via $(\mathrm{F}(3,116)=2,47 ; \mathrm{p} \leq 0.05)$, seguido pelo post-hoc de Tukey $(\mathrm{p} \leq 0,05)$ demonstrou diferença significativa no grupo de 50-59 comparado aos grupos de 20-29 e 30-39 anos. Esses indivíduos apresentaram baixa pontuação, indicando menores riscos para o desenvolvimento dos ECA. A população em estudo não apresentou pontuações acima do ponto de corte, apesar disto, de acordo com os resultados é possível verificar que conforme aumenta a idade reduzem as chances de manifestar o TCAP.

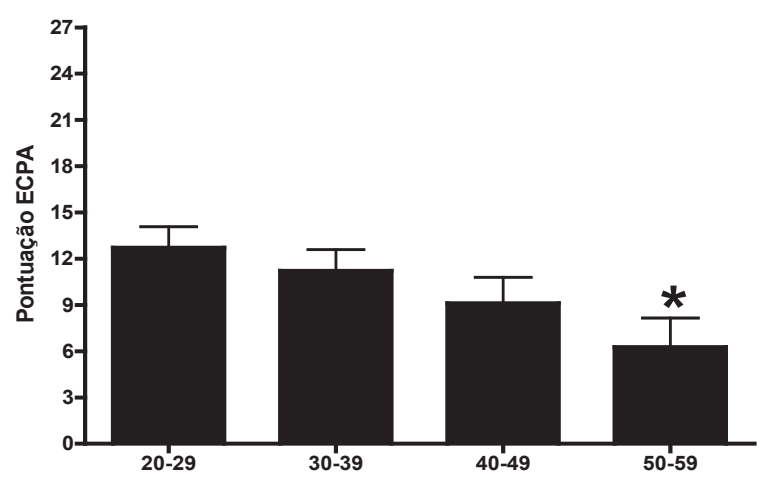

Figura 2: Escore de respostas obtidas na ECAP em indivíduos entrevistados em dois municípios do sudoeste do Paraná distribuídos de acordo com a faixa etária. Os dados expressam a média \pm EPM. ANOVA de uma via, seguida do post hoc de Tukey, ${ }^{*} \mathrm{p} \leq 0,05$, comparado aos grupos 20-29 anos e 30-39 anos.

Na figura 3 os dados foram analisados considerando as duas variáveis: estado nutricional e faixa etária. A ANOVA de duas vias revelou alterações significativas tanto para o fator estado nutricional $(\mathrm{F}(2,108)=4,72 ; \mathrm{p} \leq 0.01)$ e faixa etária $(F(3,108)=2,72 ; p 0.05)$. Nenhuma interação significativa foi encontrada $(\mathrm{F}(6,108)=0,26 ; \mathrm{p}=0.95)$. A análise com o post-hoc de Tukey demonstrou que os indivíduos obesos da faixa etária de 30-39 anos apresentaram escores mais elevados sendo significantes em relação aos indivíduos eutróficos da mesma faixa etária $(\mathrm{p} \leq 0,05)$. Observa-se que apesar dos indivíduos com 20-29 anos apresentarem pontuações mais altas nenhuma diferença significativa foi encontrada.

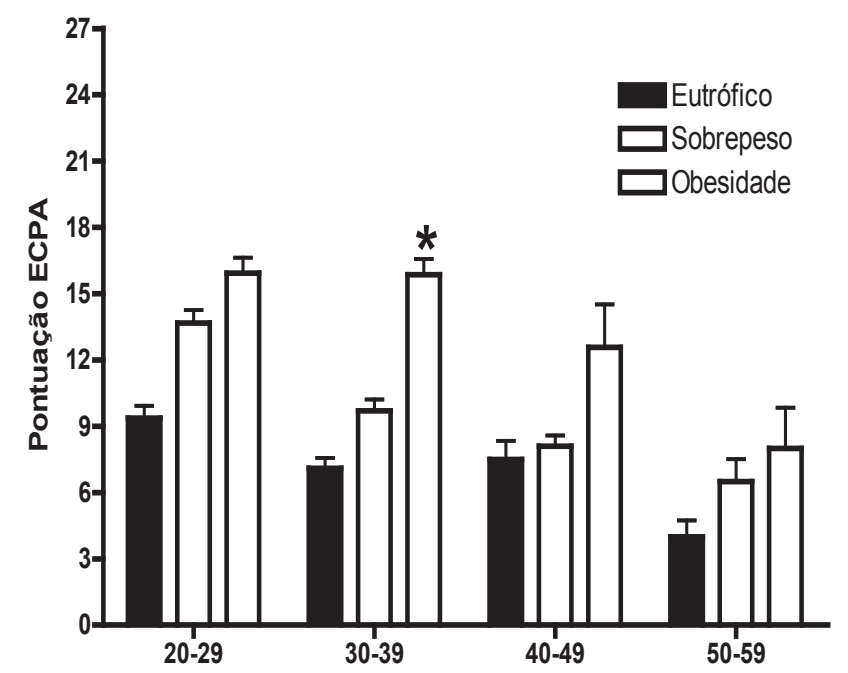

Figura 3: Escore de respostas obtidas na ECAP em indivíduos entrevistados em dois municípios do sudoeste do Paraná distribuídos de acordo com o estado nutricional e a faixa etária. Os dados expressam a média \pm EPM. ANOVA de duas via, seguida do post hoc de Tukey, $* \mathrm{p} \leq 0,05$, comparado ao grupo eutrófico da mesma faixa etária.

\section{Discussão}

A população feminina jovem (12 a 28 anos) é a mais atingida pelo TCAP, numa proporção de 20 casos em mulheres para cada caso em homens (CORDÁS, 2004). Por causa dessa diferença na prevalência entre os gêneros, os transtornos alimentares são considerados pela literatura como um problema eminentemente feminino. Isto repercute no discurso dos artigos, que, em sua maioria, referem-se à população do estudo sempre no gênero feminino (VALE, 2002). Em uma pesquisa recente realizada por Prisco et al. (2013) em trabalhadores, confirmaram-se os dados observados na literatura de que a maior prevalência de TCAP ocorre em indivíduos do sexo feminino. Portanto, neste estudo, em função dos dados observados na literatura optou-se por incluir na pesquisa, somente participantes do sexo feminino.

Os resultados obtidos no presente estudo indicam que quando os dados foram agrupados de acordo com a faixa etária, todos os sujeitos da pesquisa encontram-se, em média, abaixo do escore considerado como predisposto a desenvolver ECA ( $<17$ pontos) (figura 2). Entretanto, observam-se valores de pontuações mais elevadas na população entre 2029 anos e 30-39 anos quando comparados com os indivíduos com 50-59 anos.

É provável que adolescentes do sexo feminino sejam mais predispostas a apresentar ECA devido ao ideal de magreza imposto pela cultura ocidental que, muitas vezes, pressiona as adolescentes a supervalorizar aspectos relacionados ao tamanho corporal e peso condicionando atitudes e comportamentos alimentares danosos à saúde e que podem 
evoluir para transtornos alimentares (SOUTO; FERRO-BUCHER, 2006). Vários estudos corroboram com a teoria de que indivíduos que se encontram na faixa etária que compreende o final da adolescência e o adulto jovem estão entre as pessoas de maior risco de desenvolver este transtorno.

Em um estudo realizado em 1.209 adolescentes de Cuiabá, sendo a maioria do sexo feminino $(55,4 \%)$, com idade entre 15 e 17 anos (78,6\%), observou-se que 3,7\% se enquadraram no diagnóstico compatível com transtorno alimentar, sendo 1,8\% com TCAP. Detectou-se associação linear significativa e direta entre a prevalência de ECA e a idade do adolescente, sendo que os adolescentes com 19 anos tinham probabilidade 99\% maior de apresentar ECA do que aqueles com 14 anos. Dessa forma, na medida em que o peso do adolescente aumenta maior é a probabilidade de apresentar ECA (PIVETTA; SILVA, 2010).

No presente estudo, apesar de adolescentes (faixa etária 10-19 anos) não terem sido o foco, observa-se na amostra que os indivíduos mais jovens (faixas etárias de 20-29 e 30-39) apresentaram maiores incidências de desenvolver a ECA a partir dos dados obtidos na ECAP. É possível destacar também que a faixa etária de 50-59 (figura 2) apresentou os menores escores na ECAP, corroborando com os dados da literatura de que a prevalência é maior em indivíduos de menor faixa etária na idade adulta.

Estudo realizado em 2009 por Costa; Machado; Cordás demonstraram que mulheres com alto risco de manifestar o TCAP apresentavam média etária de 34,70 \pm 9,62 anos e $37,27 \pm 2,89 \mathrm{~kg} / \mathrm{m} 2$. Resultados semelhantes foram encontrados no nosso estudo, no qual se observou que a população de 30-39 anos, com estado nutricional de obesidade apresenta risco elevado de desenvolver TCAP (figura 3).

Apesar da população estudada, no geral, quando agrupados pelo estado nutricional, não ter apresentado quadros severos de CAP (escores abaixo de 17) verificou-se que os indivíduos considerados obesos obtiveram escores mais elevados quando comparados aos eutróficos (figura 1), sugerindo que o IMC elevado pode estar associado ao desenvolvimento de TCAP.

Resultados semelhantes foram obtidos na pesquisa de Palavras et al. (2011), no qual se observou por meio de uma revisão sistematizada que a prevalência de TCAP em estudos com obesos em programas para perda de peso foi de $16 \%$ a $51,6 \%$. A comparação entre obesos com e sem TCAP demonstrou maior tendência dos indivíduos acometidos estarem com peso mais elevado, oscilação ponderal do peso, preocupação com peso e forma corporal além da associação com comorbidades psiquiátricas (RUEDA, 2005).

Em outro estudo realizado por Matos et al. (2002) com 50 pacientes com IMC de 40 a $81,7 \mathrm{Kg} / \mathrm{m}^{2}$ (média $=52,2$ $\pm 9,2 \mathrm{Kg} / \mathrm{m}^{2}$ ) e idade entre 18 e 56 anos (média de $38,5 \pm$ $9,7)$, observou-se que a frequência de TCAP foi de $36 \%$ e $54 \%$, respectivamente. Esses indivíduos também apresentavam sintomas depressivos graves, ansiedade e preocupação com a imagem corporal, principalmente nos pacientes com obesidade grau III. Neste estudo não foi avaliado comorbidades associadas, somente foi traçado um perfil da população do sudoeste em manifestar ECA.

Embora o TCAP não se encontre limitado aos indivíduos obesos, é um diagnóstico frequentemente observado neste grupo, especialmente naqueles que procuram tra- tamento para perder peso. Enquanto a prevalência estimada de TCAP na população geral pode variar de 1,5 a 5\%, em amostras clínicas de pacientes obesos encontra-se em torno de 7,5 a 30\%. Além do mais, pacientes com TCAP usualmente evidenciam uma elevação maior do que a esperada em relação às taxas de psicopatologia alimentar (perturbações da imagem corporal) e psicopatologia geral (depressão, ansiedade e impulsividade) (APPOLINARIO, 2004). Esses dados corroboram com os resultados desta pesquisa, uma vez que os indivíduos obesos foram à população de maior risco apresentando os valores mais altos na ECAP.

Nesta pesquisa não foi avaliado o grau de satisfação corporal, entretanto diversos estudos correlacionam este fator com a presença de TCAP. Assim, percebe-se que a insatisfação corporal em mulheres obesas reflete no desenvolvimento de TCAP. O estudo de Moreira; Batista (2007) considera a insatisfação corporal como um reflexo de fatores psicopatológicos relacionados ao TCAP, tais como: baixa autoestima, depressão, preocupação extrema com a aparência, e não com o peso corporal. Neste estudo, foram avaliados 156 pacientes do sexo feminino entre 18 e 77 anos com estado nutricional de obesidade que procuraram tratamento para emagrecer, ao final do estudo identificou-se 46 pacientes com TCAP $(29,4 \%)$. Em relação à imagem corporal, mulheres obesas com TCAP apresentavam níveis significativamente mais elevados no BSQ (Body Shape Questionnaire) do que mulheres sem TCAP $(148,9 \pm 26,7$ versus $133,8 \pm 29,8$, respectivamente; $\mathrm{p}=0,003$ ) (MOREIRA; BATISTA, 2007).

Alguns estudos também correlacionam o aumento da resistência periférica à insulina (fator observado prevalentemente em indivíduos obesos), como um fator de risco para o desenvolvimento do diabetes mellitus (DM) do tipo 2 e do TCAP. Assim, o TCAP parece ser o transtorno alimentar mais encontrado entre pacientes com diabetes do tipo 2 com sobrepeso e obesidade. No entanto, na maioria dos casos, o TCAP ocorre após o diagnóstico do diabetes. Nos estudos de prevalência, observa-se que os pacientes com DM do tipo $2(59,4 \%)$ são mais acometidos com TCAP do que aqueles com DM do tipo 1 (AZEVEDO; PAPELBAUM; D'ALIA, 2002).

Portanto, o TCAP, é um TA com características ainda incertas e muitas vez pode estar associado ou ser precipitada por outras comorbidades. Diferentes estudos demonstram alguns sinais e critérios sugestivos de um diagnóstico sindrômico, contudo faltam diretrizes mais apuradas para organizar um grupo razoavelmente homogêneo e caracterizá-lo como categoria diagnóstica (AZEVEDO, SANTOS; FONSECA, 2004). Assim, o instrumento aplicado neste estudo, a ECAP (FREITAS et al., 2001) apresenta-se como uma ferramenta útil e de fácil aplicação para estudos epidemiológicos de maior abrangência, com o objetivo de identificar os indivíduos de risco. Entretanto, torna-se evidente, a partir dos resultados que outros estudos são requeridos para obter-se uma amostra representativa da população do sudoeste e verificar o perfil de risco de TCAP associado às comorbidades correlacionadas.

Por fim, é necessário bom senso e compreensão da doença, bem como instrumentos rápidos para verificar populações de risco e profissionais habilitados para aplicá-lo. O nutricionista deve compor a equipe multiprofissional, pois a partir da identificação este profissional estará habilitado para 
propor modificações na dieta e hábitos alimentares dos indivíduos acometidos.

\section{Conclusão}

Os resultados do presente estudo demonstram que, na média da amostra avaliada, os participantes apresentaram escores abaixo dos valores considerados como risco para desenvolvimento do TCAP, a partir do instrumento avaliado (ECAP), entretanto foi possível verificar que o estado nutricional contribui para elevar este risco, uma vez que os indivíduos com obesidade apresentaram escores elevados significativos comparados aos eutróficos.

Diante das reflexões e dos achados do presente estudo, percebe-se que a grande maioria $(80 \%)$ desses indivíduos não apresentou CAP. Contudo os indivíduos com IMC maior apresentaram escores na ECAP mais elevados, refletindo maiores riscos na população com obesidade, também pode-se observar que os indivíduos entre 20-39 anos apresentaram pontuação mais elevada, comparando aos indivíduos de 50-59 anos em que é possível verificar que conforme aumenta a idade reduzem as chances de manifestar o TCAP; portanto, sugerem-se mais estudos com esta população alvo, averiguando se o estado nutricional estaria relacionado com a CAP, para assim esclarecer melhor a relação que esses sujeitos possuem com o TCAP.

\section{Referências}

AZEVEDO, A. P.; SANTOS, C. C.; FONSECA, D. C. Transtorno da compulsão alimentar periódica. Revista de Psiquiatria Clínica, São Paulo, v. 31, n. 4, p. 170-172, set. 2004.

AZEVEDO, A. P.; PAPELBAUM, M.; D’ALIA, F. Diabetes e transtornos alimentares: uma associação de alto risco.

Revista Brasileira de Psiquiatria, Rio de janeiro, v. 24, n. 3, p. 77-80, dez. 2002.

APPOLINARIO, J. C. Transtorno da compulsão alimentar periódica: uma entidade clínica emergente que responde ao tratamento farmacológico. Revista Brasileira de Psiquiatria, Rio de Janeiro, v. 26, n. 2, p. 75-76, jun. 2004.

AMERICAN Psichiatric Association. Diagnostic and Statistical Manual of Mental Disorders (DSM IV). 4 ed. Washinton: Artmed, 1994.

BARBETTA, P. A. Estatística aplicada as Ciências Sociais. 8 ed. Florianópolis: UFSC, 2012.

BENOIT, S. C. et al. Novel functions of orexigenic hypothalamic peptides: from genes to behavior. Nutrition, v. 24, p. 843-847, sept. 2008.

CENCI, M.; PERES, K. G.; VASCONCELOS, F. A. G. Prevalência de comportamento bulímico e fatores associados em universitárias. Revista de Psiquiatria Clínica, São Paulo, v. 36, n. 3, p. 83-88, fev. 2009.
Imagem corporal e comportamento sexual de mulheres obesas com e sem transtorno da compulsão alimentar periódica. Revista de Psiquiatria Clínica, São Paulo, v. 37 , n. 1, p. 27-31, abr. 2009.

CORDÁS, T.A. Transtornos Alimentares: classificação e diagnóstico. Revista de Psiquiatria Clínica, v. 31 , n. 4 , p. 154-157, 2004.

CORTEZ, C. M.; ARAÚJO, E. A.; RIBEIRO, M. V. Transtorno da compulsão alimentar periódico e obesidade. Arquivos Catarinenses de Medicina, Rio de Janeiro, v. 40, n. 1, p. 94-102, set. 2011.

DUCHESNE, M.; ALMEIDA, P. E. M. Terapia cognitivocomportamental dos transtornos alimentares. Revista Brasileira de Psiquiatria, São Paulo, v. 24, n. 3, p. 49-53, dez. 2002.

DEVASKAR, S. V. Neurohumoral Regulation of body weight gain. Pediatric Diabetes, v. 2, n. 2, p. 131-144, Sept. 2001.

FREITAS, S. et al. Tradução e adaptação para o português da Escala de Compulsão Alimentar Periódica. Revista Brasileira de Psiquiatria, Rio de Janeiro, v. 23, n. 4, p. 215-220, nov. 2001.

GORMALLY, J. et al. The assessment of binge eating severity among obese persons. Addict Bebav, New York, v. 7, n.1, p. 47-55, sept. 1982.

GONÇALVES, M. Transtorno compulsivo alimentar periódico. Disponível em: <http://www.polbr.med.br/ ano12/prat1012.php $>$. Acessado em: 05/11/2013.

KAYE, W. H.; WELTZIN, T. E. Neurochemistry of bulimia nervosa. Journal of Clinical Psychiatry, Europe, v. 52, n. 21, p. 21-28, sep. 1991.

LATTERZA, A. R. et al. Tratamento nutricional dos transtornos alimentares. Revista de Psiquiatria Clínica, São Paulo, v. 31, n. 4, p. 173-176, set. 2004.

MATOS, M. I. R. et al. Binge eating disorder, anxiety, depression and body image in grade III obesity patients. Revista Brasileira de Psiquiatria, São Paulo, v. 24, n. 4, p. 165-169, jul. 2002.

MONTELEONE, P. et al. Serotonergic dysfunction across the eating disorders: relationship to eating behaviour, purging behaviour, nutritional status and general psychopathology. Psychological Medicine, Italy, v. 30, p. 1099-110, oct. 2000.

MOREIRA, R. O.; BATISTA, A. P. Insatisfação com a imagem corporal em mulheres obesas: a importância do transtorno da compulsão alimentar periódica. Revista de Psiquiatria do Rio Grande do Sul, Rio de Janeiro, v. 29, n. 1, p. 130-131, jan. 2007.

COSTA, R. F.; MACHADO, S. C.; CORDÁS, T. A. 
MUNSCH, S.; BEGLINGER, C. Obesity and Binge

Eating Disorder. Washington: Karger, 2005.

NUNES, M. R. Transtorno da compulsão alimentar periódica e a abordagem da terapia cognitiva comportamental. 2012. 23 f. Dissertação (Especialização em Nutrição) - Universidade Federal de Juiz de Fora, Juiz de Fora, 2012.

PALAVRAS, M. A. et al. Uma revisão dos estudos latinoamericanos sobre o transtorno da compulsão alimentar periódica. Revista Brasileira de Psiquiatria, São Paulo, v. 33, n. 1, mai. 2011.

PASSOS, T. C.; STEFANO, S. C.; BORGES, M. B. Transtornos Alimentares e Obesidade. 2 edição. São Paulo: Manole, 2005.

PAPELBAUM, M.; APPOLINÁRIO, J. C. Transtorno da compulsão alimentar periódica e tra nstorno obsessivocompulsivo: partes de um mesmo espectro?. Revista Brasileira de Psiquiatria, São Paulo, v. 23, n. 1, p. 38-40, dez. 2001.

PAVLOVA, I. V.; VANETSUAN, G. L. Evoked activity of the cat hypothalamus and amygdale under food motivation and in emotional stress. Ross Fiziol Zh Im I M Sechenova, Russian, v. 90, p. 1472-1484, dec. 2004.

PIVETTA, L. A.; SILVA, R. M. Compulsão alimentar e fatores associados em adolescentes de Cuiabá, Mato Grosso, Brasil. Caderno de Saúde Pública, Rio de Janeiro, v. 26, n. 2, p. 337-346, fev. 2010.

PRISCO, A. P. et al. Prevalência de transtornos alimentares em trabalhadores urbanos de município do Nordeste do Brasil. Ciência \& Saúde Coletiva, Bahia, v. 18, n. 4, p. 1109-1118, out. 2013.

RICCA, V. et al. Cognitive-behavioural therapy for bulimia nervosa and binge eating disorder: a review. Psychother Psychosom, Glasgow, v. 69, n. 3, p. 287-295, may. 2000.

RUEDA, J. G. Trastorno por atracones: ¿entre los trastornos de la conducta alimenticia y la obesidad? Una mirada cognoscitiva comportamental. Revista Colombiana de Psiquiatría, Colombia, v. 19, n. 2, jun. 2005.

SANTANA, M. L. P. et al. La epidemiología y los factores de riesgo de los trastornos alimentarios en la adolescencia; una revisión. Nutrición Hospitalaria, Madrid, v. 27, n. 2, p. 391-401, abr. 2012.

SALDAÑA, C. Tratamientos psicológicos eficases para Trastornos del comportamiento alimetario. Psicothema, Barcelona, v. 13, n. 3, p. 381-392, mar. 2001.

SÁNCHEZ, J. C. Perfil fisiológico de la leptina. Colombia Médica, Colombia, v. 36, n. 1, p. 50-59, jun. 2005.

STEFANO, S. C.; BORGES, M. B. F; CLAUdINO, A. M.
Guia Prático de Psiquiatria clínica. São Paulo: Manole, 2002, p. 121-126.

SPITZER, R.L. et al. Binge Eating Disorder: its further validation in a multisite study. 13 ed. New York: Copyright, 1993. p. 137-153.

SOUTO, S.; FERRO-BUCHER, J. S. N. Práticas indiscriminadas de dietas de emagrecimento e o desenvolvimento de transtornos alimentares. Revista de Nutrição, Campinas, v. 19, n. 6, p. 693-704, dez. 2006.

VALE, A. M. Comportamento alimentar anormal e práticas alimentares inadequadas para controle de peso entre adolescentes do sexo feminino de fortaleza. 2002. 129 f. Dissertação (Mestrado em Saúde Pública) Universidade Federal do Ceará, Fortaleza, 2002.

WORLD HEALTH ORGANIZATION (WHO). Physical status: the use and interpretation of anthropometry. Disponível em: < http://www.who.int/childgrowth/ publications/physical_status/en/>. Acessado em: $10 \mathrm{de}$ Outubro. 2013. 